https://doi.org/10.15407/ujpe65.11.958

B.E. GRINYUK, I.V. SIMENOG

Bogolyubov Institute for Theoretical Physics, Nat. Acad. of Sci. of Ukraine

(14-B, Metrolohychna Str., Kyiv 03143, Ukraine; e-mail: bgrinyuk@bitp.kiev.ua)

\title{
ON THE TEMPERATURE ROLE IN THE TUNNELING PROCESS AT THE LOW-ENERGY NUCLEAR FUSION
}

\begin{abstract}
The temperature dependence of the coefficient of tunneling through the Coulomb barrier is estimated for nuclei of the hydrogen isotopes at comparatively low temperatures using a model of screened Coulomb interaction potential between the isotopes put inside an external oscillator potential well. The temperature dependences for the tunneling coefficient are calculated for pp-, $p d-, p t-, d d-$, and $d t$-processes at different screening radii. The probable role of pp-reactions is discussed.

Keywords: low-energy nuclear fusion, tunneling through the screened Coulomb barrier, temperature dependence of the tunneling rate
\end{abstract}

\section{Introduction}

Nuclear reactions are generally known to be independent of the temperature within our usual temperature scale. But this is not the case of nuclear fusion reactions connected with the tunneling process through a Coulomb barrier. In the present paper, we consider a simple model of two nuclei of hydrogen isotopes settled into a crystal defect, and we demonstrate an essential dependence of the fusion reaction rate on the temperature at its rather low values.

Although the probability of the tunneling through a Coulomb barrier is too small in order to speculate about a possibility of the "cold fusion" and its practical usage, but the problem is still actual [1] and is of interest from the general theoretical point of view. In particular, we will consider the dependence of the transparency of a screened Coulomb barrier on such natural and easily changeable physical parameter, as the temperature varies not within the range of millions of degrees, but from $0 \mathrm{~K}$ to the crystal melting point (for definiteness, we take $2000 \mathrm{~K}$ ). This study is mainly based on [2]. The below-discussed effect of an increase in the barrier transparency, as the temperature grows, is rather close in some sense to the tunneling rate increasing due to fluctuations [3]. We do not discuss the important role of electrons in fusion reactions, in the excitation of nuclei [4], and even in decay reactions [5] in detail. But in the view of possible ef-

(C) B.E. GRINYUK, I.V. SIMENOG, 2020

958 fects of an additional barrier screening inside a defect with high electron density, we find the temperature dependences at different screening radii (from $0.5 \AA$ down to $0.1 \AA$ ). We also consider different masses (for the $p p-, p d-, p t-, d d-$, and $d t$-reactions).

\section{A Model of Two Nuclei of Hydrogen Isotopes in a Crystal Defect}

We consider a phenomenological Hamiltonian describing the system of two point-like nuclei of masses $m_{1}$ and $m_{2}$ in the region of the crystal defect in the following form:

$$
\begin{aligned}
& \hat{H}=-\frac{\hbar^{2}}{2 m_{1}} \triangle_{1}-\frac{\hbar^{2}}{2 m_{2}} \triangle_{2}+V_{\text {nucl }}\left(\left|\mathbf{r}_{\mathbf{1}}-\mathbf{r}_{\mathbf{2}}\right|\right)+ \\
& +\frac{e^{2}}{\left|\mathbf{r}_{\mathbf{1}}-\mathbf{r}_{\mathbf{2}}\right|} \exp \left(-\frac{\left|\mathbf{r}_{\mathbf{1}}-\mathbf{r}_{\mathbf{2}}\right|}{r_{\mathrm{D}}}\right)+W\left(r_{1}, r_{2}\right),
\end{aligned}
$$

where the screening of the Coulomb repulsion between the nuclei is taken into account in a standard way $\left(r_{\mathrm{D}}\right.$ is the Debye screening radius in the defect area). The presence of a defect is taken into account simply by introducing an external spherically symmetric potential $W$. A specific form of the external field is not too important for the tunneling process through the screened Coulomb potential. For convenience, in view of the further separation of variables, we use it in the form

$W\left(r_{1}, r_{2}\right)=K \cdot\left(\lambda_{1} r_{1}^{2}+\lambda_{2} r_{2}^{2}\right)$

ISSN 2071-0194. Ukr. J. Phys. 2020. Vol. 65, No. 11 
with $\lambda_{1}=m_{1} /\left(m_{1}+m_{2}\right)$ and $\lambda_{2}=m_{2} /\left(m_{1}+m_{2}\right)$. For equal masses, one obviously has $\lambda_{1}=\lambda_{2}=1 / 2$. For a potential well typical of a defect of the order of $\sim 1 \mathrm{eV}$ in depth and about $4 \cdot 10^{-8} \mathrm{~cm}$ in radius, one has $K \sim 10^{15} \mathrm{eV} \cdot \mathrm{cm}^{-2}$. As to the nuclear interaction between the two particles $V_{\text {nucl }}$, we only use the fact of the short-range character of nuclear forces (their radius is known to be of the order of $\sim 10^{-13} \mathrm{~cm}$ ).

As it is clear a priori (see Fig. 1) and shown by calculations [2], the last term in (1) does not make an essential influence on the tunneling rate at the room and higher temperatures, i.e. a particular form of the last term in (1) is not too important. More important is the influence of the screening radius depending on the concentration of negative particles (electrons present in crystal or specially injected into it in some way). Much better for the screening effect are known to be $\mu_{e}^{-}$-mesons resulting in the screening radii of about $\frac{m_{\mu}}{m_{e}} \cong 207$ times less than those due to electrons. But here we do not dwell upon a separate important problem of $\mu$-catalysis restricting ourselves with $r_{\mathrm{D}}$ of the order of $0.1-0.5 \AA$ typical of ordinary crystals with electrons.

An advantage of the Hamiltonian in the form (1) with the potential well (2) lies in the fact that the center-of-mass variable can be separated explicitly giving rise to a one-variable problem with the Hamiltonian

$\hat{h}=-\frac{\hbar^{2}}{2 \mu} \Delta+V_{\text {nucl }}(r)+\frac{e^{2}}{r} \exp \left(-\frac{r}{r_{\mathrm{D}}}\right)+\frac{1}{2} \kappa r^{2}$,

where $r \equiv\left|\mathbf{r}_{\mathbf{2}}-\mathbf{r}_{\mathbf{1}}\right|$, the reduced mass $\mu \equiv$ $\equiv m_{1} m_{2} /\left(m_{1}+m_{2}\right)$, and $\kappa \equiv \frac{2 m_{1} m_{2}}{\left(m_{1}+m_{2}\right)^{2}} K$. Moreover, since we are going to study the tunneling process through the barrier like the screened Coulomb one, we may restrict ourselves with the s-state solutions of the problem, because the additional centrifugal barrier $\sim \frac{l(l+1)}{r^{2}}$ typical of the $l \neq 0$ states makes the tunneling probability negligible for these states. For the s-states, one can use the well-known notation $u(r) \equiv r \psi(r)$ and come to a one-dimensional problem

$-\frac{\hbar^{2}}{2 \mu} u^{\prime \prime}(r)+\left(V_{\text {nucl }}(r)+\frac{e^{2}}{r} \exp \left(-\frac{r}{r_{\mathrm{D}}}\right)+\frac{1}{2} \kappa r^{2}\right) u(r)=$
$=E u(r)$.

Further, we consider the tunneling process through the central repulsive part of a potential

$V(r)=V_{\text {nucl }}(r)+\frac{e^{2}}{r} \exp \left(-\frac{r}{r_{\mathrm{D}}}\right)+\frac{1}{2} \kappa r^{2}$

ISSN 2071-0194. Ukr. J. Phys. 2020. Vol. 65, No. 11

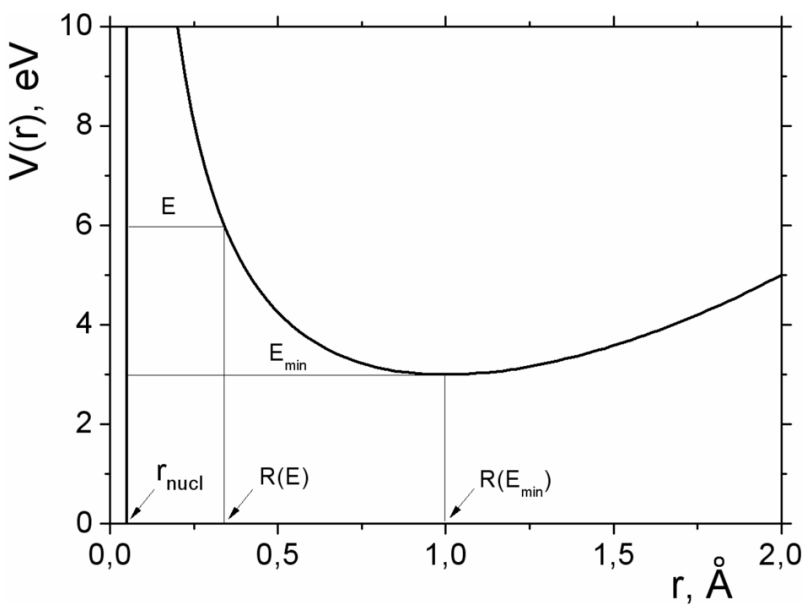

Fig. 1. A schematic profile of the potential $V(r)$

(see Fig. 1) within the well-known quasiclassical approximation. We also consider a subsidiary Hamiltonian

$\stackrel{\hat{h}}{=}=-\frac{\hbar^{2}}{2 \mu} \frac{d^{2}}{d r^{2}}+\frac{e^{2}}{r} \exp \left(-\frac{r}{r_{\mathrm{D}}}\right)+\frac{1}{2} \kappa r^{2}$,

where the nuclear potential is omitted. If one uses the dimensionless units for the coordinate $x=r / r_{\mathrm{D}}$ and for the energy $\mathcal{E}=\frac{\mu r_{\mathrm{D}}^{2}}{\hbar^{2}} E$, the Hamiltonian (6) becomes dependent on two parameters only (they are the intensities of the screened Coulomb potential and the oscillator potential well). This trivial fact may be useful for a general analysis of the problem. But we keep further the original form of Hamiltonian (6) which may seem to contain a greater number of parameters.

\section{Temperature Enhancement of the Barrier Transparency}

The transparency coefficient for the tunneling process through a potential barrier is known to be (see, e.g., [6])

$D(E) \simeq \exp \left(-\frac{2}{\hbar} \int_{r_{\text {nucl }}}^{R(E)} \sqrt{2 \mu(V(r)-E)} d r\right)$

where the integral in the exponent essentially depends on the relative energy $E$ of colliding particles. The general multiplier of the order of 1 is neglected. The effect of the barrier transparency increasing with the temperature growth is based on the fact that, at nonzero temperature, there exists a nonzero probability for particles to collide with energies greater than 


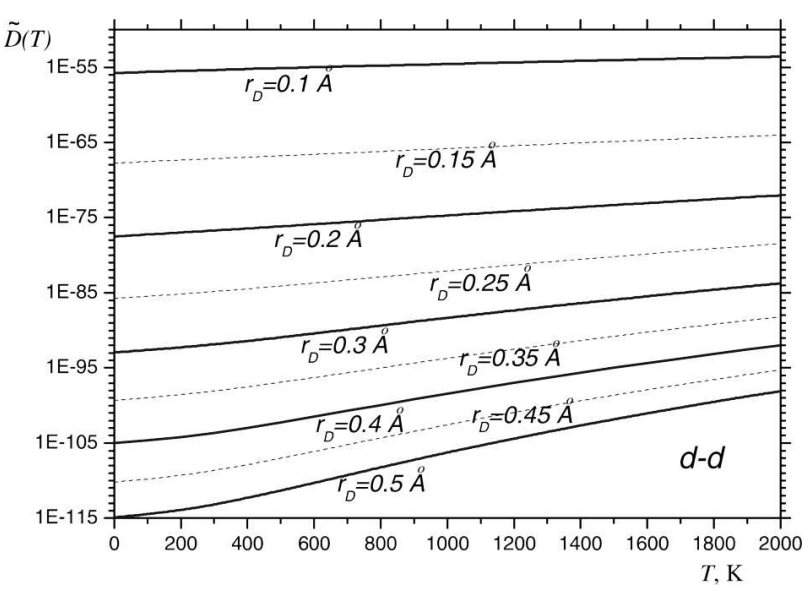

Fig. 2. Temperature dependence of $\widetilde{D}$ for the $d d$-tunneling at different screening radii

their ground state energy $E_{0}$, i.e. to pass through the barrier much easier. Although the above probability decreases exponentially, as the energy increases, due to the Boltzmann factor $\sim \exp \left(-\left(E-E_{0}\right) / k T\right)$, the increase of the transparency coefficient appears to be considerably greater giving rise to the total growth of the tunneling rate. We can introduce an effective transparency coefficient $\widetilde{D}(T)$ to be the average

$\widetilde{D}(T)=\frac{\sum_{n} D\left(E_{n}\right) \exp \left(-\frac{E_{n}-E_{0}}{k T}\right)}{\sum_{n} \exp \left(-\frac{E_{n}-E_{0}}{k T}\right)}$,

where the summation is spread over all the states of Hamiltonian (6) having the corresponding energies $E_{n}$. Since we deal with a reduced one-dimensional Hamiltonian, the degeneracy $g_{n}$ of the states equals 1 , and that is why $g_{n}$ is not shown explicitly in (8).

To simplify the further calculations, we replace the summation in (8) by the integration using the fact that the discrete energy levels of the system are rather close one to another because of a comparatively large reduced mass $\mu$ of nuclei:

$\widetilde{D}(T) \rightarrow \frac{\int_{\min }^{\infty} D(E) \exp \left(-\frac{E-E_{\min }}{k T}\right) \lambda(E) d E}{\int_{E_{\min }}^{\infty} \exp \left(-\frac{E-E_{\min }}{k T}\right) \lambda(E) d E}$,

where $E_{\text {min }}$ is the minimal energy (see Fig. 1). The transition from (8) to (9) demands us to know the density of energy states $\lambda(E)$. But, in our model, this smooth function varies less than within one order of magnitude. Using the mean-value theorem and taking $\lambda\left(E^{*}\right)$ out from the integrand of the numerator in (9), and $\lambda\left(E^{* *}\right)$ from the integrand of the denominator, and ignoring the multiplier $\frac{\lambda\left(E^{*}\right)}{\lambda\left(E^{* *}\right)}$ being of the order of magnitude of $\sim 10^{0}$, we come to the approximate relation

$\widetilde{D}(T) \sim \frac{\int_{\min }^{\infty} D(E) \exp \left(-\frac{E-E_{\min }}{k T}\right) d E}{\int_{E_{\min }}^{\infty} \exp \left(-\frac{E-E_{\min }}{k T}\right) d E}$,

which can be easily computed.

It is suitable to depict the temperature dependences for $\widetilde{D}(T)$ on the logarithmic scale. Figure 2 shows such dependences in the case of $d d$ fusion for different screening radii, at fixed $\kappa=$ $10^{-17} \mathrm{MeV} \cdot \mathrm{fm}^{-2}$, and $r_{\text {nucl }}=2 \mathrm{fm}$. Generally, the temperature dependence of the tunneling (and, hence, the fusion) rate is seen to be essential: $\widetilde{D}(T)$ is increasing at least a few orders of magnitude under the temperature change within about $\sim 10^{2}-10^{3}$ degrees. Thus, the temperature effect is essential and should be taken into account in all the estimates and calculations concerning the problem of low-energy nuclear fusion.

\section{Dependence \\ of the Effective Transparency Coefficient on the Reduced Mass}

The tunneling rate is known to be dependent on the mass of a particle penetrating though a potential barrier. In our case of two nuclei, as is seen from (7), the exponent is proportional to the square root from the reduced mass of these nuclei. For the effective transparency coefficient $\widetilde{D}$, the dependence on $\mu$ is very similar: the less the reduced mass, the greater is $\widetilde{D}$. This fact is illustrated by Fig. 3, where the dependence of $\widetilde{D}$ on the reduced mass $\mu$ is shown. For definiteness, we take $r_{\mathrm{D}}=0.5 \AA$, and depict curves for three temperature values: $T=0 \mathrm{~K}, T=1000 \mathrm{~K}$, and $T=2000 \mathrm{~K}$. On the bottom of the figure, specific values of $\mu$ are depicted for different pairs of hydrogen isotopes.

Consider the cases of different pairs of isotopes in detail. In Fig. 4, we show the temperature dependence of $\widetilde{D}(T)$ for the $p p$-tunneling, where one can see an essentially greater transparency coefficients as compared to the $d d$-tunneling (Fig. 2). Similar temperature dependences of $\widetilde{D}(T)$ for the $p d-, p t-$, and

ISSN 2071-0194. Ukr. J. Phys. 2020. Vol. 65, No. 11 


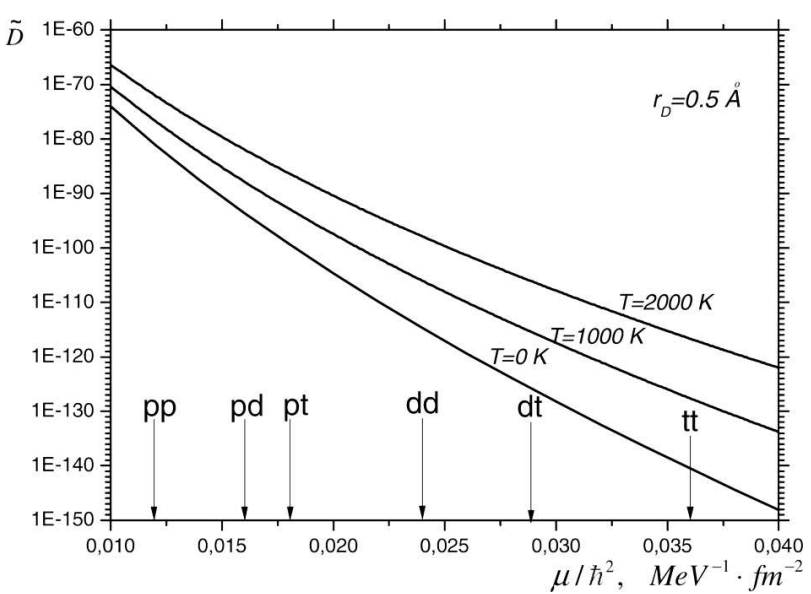

Fig. 3. Dependence of $\widetilde{D}$ on the reduced mass $\mu$

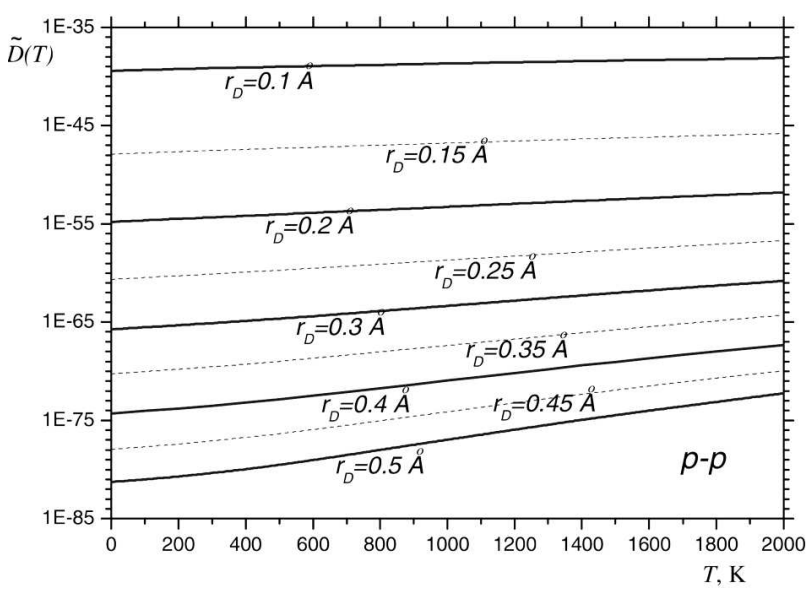

Fig. 4. Temperature dependence of $\widetilde{D}$ for the $p p$-tunneling

$d t$-tunnelings confirm the general conclusion about the dependence of $\widetilde{D}$ on the reduced mass and are shown in Figs. 5, 6, and 7, respectively.

In all the considered cases, the essential role of temperature in the tunneling is confirmed being at least a few orders of magnitude of $\widetilde{D}$ growth with the temperature increase up to hundreds of degrees. Thus the rate of consequent nuclear reactions is essentially dependent on the temperature, though this rate remains to be very low for the practical usage.

\section{About the Possible Role of $p p$-Reactions in the Low-Energy Nuclear Fusion}

A number of reactions per second in a sample with defects containing isotopes of hydrogen is evidently ISSN 2071-0194. Ukr. J. Phys. 2020. Vol. 65, No. 11

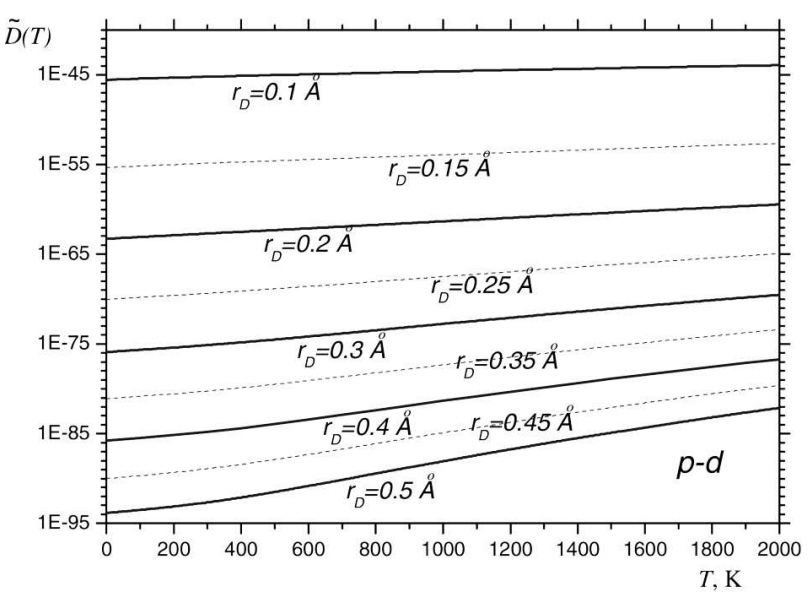

Fig. 5. The $\widetilde{D}$ temperature dependence in the case of $p d$ tunneling

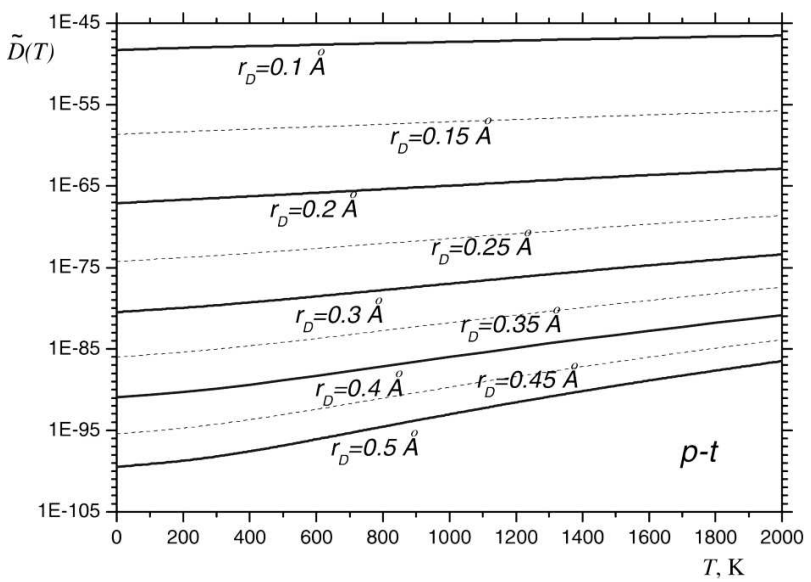

Fig. 6. Effective transparency $\widetilde{D}$ versus the temperature for the $p t$-tunneling

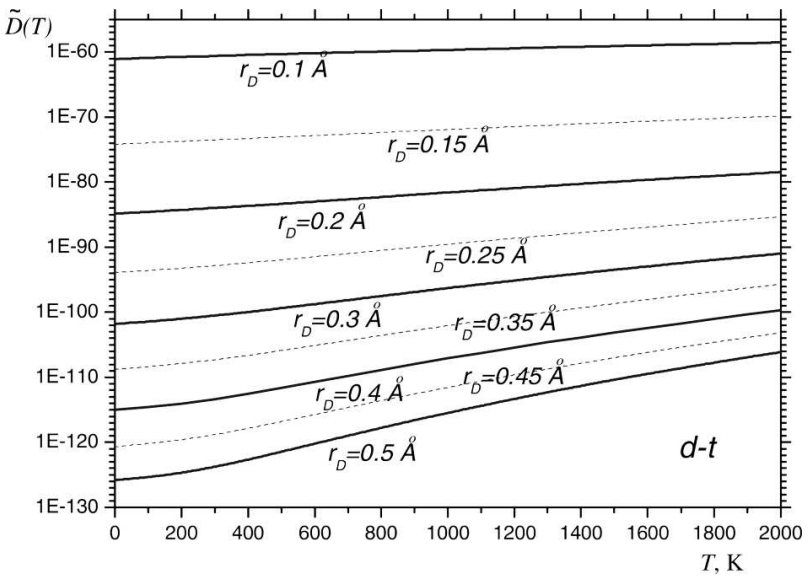

Fig. 7. $\widetilde{D}(T)$ for the $d t$-tunneling 
proportional to the number of these defects. Let $\nu=$ $\sqrt{\kappa / \mu}$ be the frequency of the oscillator potential $\frac{1}{2} \kappa r^{2}$ in Hamiltonian (5). Typical value of $\nu$ in our case is of the order of $10^{13} \mathrm{sec}^{-1}$. Within a quasiclassical approach, this frequency has a value of the order of a number of collisions of two hydrogen isotopes in a defect per second. If one had even $N_{0} \sim 10^{20}$ defects in each gram, the number of reactions of nuclear fusion per second in a gram, of the order of $N_{0} \cdot \nu \cdot \widetilde{D}$, would be too small due to the smallness of $\widetilde{D}$ (even for $r_{\mathrm{D}} \sim 0.1 \AA$, see Figs. 2, 4-7). But for the $p p$-tunneling, this small probability is obviously the highest one, due to the smallest reduced mass, of course.

Thus, the most probable reaction in a sample with defects saturated by isotopes of hydrogen is the $p p$-reaction resulting in $p+p \rightarrow d+e^{+}+$ $+\nu_{e}+0.42 \mathrm{MeV}$. The consequent annihilation of the positron in a media with electrons gives an additional energy of $2 m_{e} c^{2} \cong 1.02 \mathrm{MeV}$ in the form of $\gamma$-quanta. It is important that the deuteron obtained in this reaction has an energy $\leq 0.08 \mathrm{MeV}$ (or $\sim 10^{9} \mathrm{~K}$ ) that is much greater than the temperature of the crystal, and this may be a source of fast deuterons for secondary $d p$-reactions (as well as for $d d$-ones, if deuterium is present in the crystal in addition to hydrogen atoms). A kinetic energy of the positron and the $\gamma$-quantum energy may also be partially transformed into the kinetic energy of nuclei of hydrogen isotopes and increase the probability of secondary reactions. Thus, the $p p$-reactions may be of importance both by themselves (giving totally about $0.42+1.02=1.44 \mathrm{MeV}$ per each reaction) and as a source of fast deuterons. We do not discuss here much less probable reaction $p+p+e^{-} \rightarrow d+\nu_{e}+1.44 \mathrm{MeV}$, which is known to take place with protons involved too. We only note that it yields the same energy output, and also gives fast deuterons.

\section{Conclusions}

To summarize, we note a noticeable influence of the temperature on the tunneling process, and this effect should be necessarily taken into account when studying the low-energy fusion reactions with hydrogen isotopes involved, although the temperature of the order of $\sim 10^{3} \mathrm{~K}$ is not high on the scale of thermonuclear reactions. With regarad for the role of fluc- tuations in stimulating the tunneling processes [3], one can obtain a little bit greater tunneling rate, because both effects are working together in the same direction. Accounting for an increase in the tunneling rate due to a screening effect (i.e. due to a decrease in $r_{\mathrm{D}}$ ), a saturation of the sample with injected electrons might be important to study. Regarding the above-discussed possible role of the most probable $p p$ reactions (both in view of the energy balance and as an "acceleration mechanism" for deuterons obtained in these reactions), it is significant to study the tunneling processes in crystals with defects containing a mixture of isotopes of hydrogen and to identify possible $p p$-reactions.

This work is supported by the National Academy of Sciences of Ukraine (Project No. 01172U000237).

1. C.P. Berlinguette, Yet-Ming Chiang, J. N. Munday, T. Schenkel, D.K. Fork, R. Koningstein, M.D. Trevithick. Revisiting the cold case of cold fusion. Nature 570 (7759), 45 (2019).

2. B.E. Grinyuk, I.V. Simenog. On the temperature role in the tunneling process at the "cold fusion". Preprint ITP-91-25E, Kyiv, 1991, 13 p.

3. M.V. Altaiiskii, S.N. Artukha, B.I. Barts, V.G. Bar'yakhtar, S.S. Moiseev. The fluctuation increase in the transparency of quantum-mechanical and wave barriers and some physical consequences. Ukr. Fiz. Zh. 35, No. 2, 297 (1990).

4. A.Ya. Dzyublik, G. Gosselin, V. Mèot, and P. Morel. Role of screening in Coulomb excitation of nuclei by electrons in hot plasma. EPL 102, 62001 (2013).

5. A.Yu. Dzyublik. Influence of electronic environment on $\alpha$ decay. Phys. Rev. C 90, 054619 (2014).

6. O.S. Davydov. Quantum Mechanics (Akademperiodyka, 2013), (in Ukrainian), [ISBN: 978-966-360-211-0].

Received 28.06.20

\section{Б.С. Гринюк, І.В. Сименог}

РОЛЬ ТЕМПЕРАТУРИ У ПРОЦЕСІ

ТУНЕЛЮВАННЯ ПРИ ХОЛОДНОМУ СИНТЕЗІ

$\mathrm{P}$ е $з$ ю м е

Проведено оцінку температурної залежності коефіцієнта тунелювання крізь кулонівський ба'рєр для ізотопів гідрогену при відносно низьких температурах у рамках моделі екранованого кулонівського потенціалу взаємодії між цими ізотопами, поміщеними у зовнішню осциляторну потенціальну яму. Температурні залежності для коефіцієнта тунелювання розраховано для $p p-, p d-, p t-, d d$ - і $d t$-реакцій при різних радіусах екранування. Обговорюється можлива роль $p p$-реакцій. 